\title{
Direct constraints on charged excitations of dark matter
}

\author{
Haipeng An, ${ }^{1}$ Maxim Pospelov, ${ }^{1,2}$ and Josef Pradler ${ }^{3}$ \\ ${ }^{1}$ Perimeter Institute, Waterloo, Ontario N2L 2Y5, Canada \\ ${ }^{2}$ Department of Physics and Astronomy, University of Victoria, Victoria, BC, V8P 5C2 Canada \\ ${ }^{3}$ Department of Physics and Astronomy, Johns Hopkins University, Baltimore, MD 21210, USA
}

\begin{abstract}
If the neutral component of weak-scale dark matter is accompanied by a charged excitation separated by a mass gap of less than $\sim 20 \mathrm{MeV}$, WIMPs can form stable bound states with nuclei. We show that the recent progress in experiments searching for neutrinoless double-beta decay sets the first direct constraint on the exoergic reaction of WIMP-nucleus bound state formation. We calculate the rate for such process in representative models and show that the double-beta decay experiments provide unique sensitivity to a large fraction of parameter space of the WIMP doublet model, complementary to constraints imposed by cosmology and direct collider searches.
\end{abstract}

Introduction Weakly interacting massive particles (WIMPs) are well-motivated candidates for dark matter (DM), that offer a variety of potential non-gravitational signatures, including the possibility for a laboratory detection. Model realizations of WIMP DM often entail multiple states. Frequently encountered are scenarios in which DM $\left(X^{0}\right)$ is part of a multiplet with an electrically charged excited state $\left(X^{ \pm}\right)$that enables to naturally regulate its abundance through co-annihilation. As has been noted in [1], if the mass gap between the neutral and charged state is sufficiently small, $M e V$-scale energy depositions become possible via the process of WIMPnucleus bound state formation.

Denote by $\left(N X^{-}\right)$the bound state of DM with a target nucleus $N$. Depending on the relation between spins of $X^{0}$ and $X^{-}$two generic scenarios can be envisaged,

$$
\begin{array}{ll}
\text { Case A: } & N_{Z}+X^{0} \rightarrow\left(N_{Z} X^{-}\right)+e^{+}, \\
\text {Case B: } & N_{Z}+X^{0} \rightarrow\left(N_{Z+1} X^{-}\right)
\end{array}
$$

where $Z$ denotes the charge of $N$. The Feynman diagrams of these two scenarios are shown in Fig. 1. If $\left(N X^{-}\right)$is not in its ground state, it will de-excite by emitting $\gamma$-rays. Thus, the observables for these processes are the positron and $\gamma$-rays (and a residual anomalously heavy nucleus.) The recombination is guaranteed to happen once the $\left(N X^{-}\right)$Coulomb binding energy $E_{b}$ allows to bridge the mass gap $\Delta m \equiv m_{X^{-}}-m_{X^{0}}$ in the DM multiplet. Mass splittings of $\sim 20 \mathrm{MeV}$ or less can be probed through (1) and (2). Case B can arise in the scalar DM models, multiplets of the SM weak group [2], with $\sim 20 \%$ cancellation between tree-level and loop-induced $\Delta m$. Both scenarios are readily realized in supersymmetric (SUSY) scenarios.

Recent FERMI-LAT observations [3] of a $135 \mathrm{GeV}$ photon excess from the galactic center stimulate studies of WIMP models with mass splitting on the order of few MeV and less, as a source of enhancement of WIMP annihilation to mono-energetic $\gamma$-rays [1]. Additional interest to models with $O(\mathrm{MeV})$ mass splitting comes from the possibility of DM catalysis of primordial nuclear reactions, changing the outcome for lithium isotopes, that currently shows unexpected deviations [4].

The primary purpose of this letter is to show that the recent advances in neutrinoless double-beta $(0 \nu \beta \beta)$ decay experiments allow for a direct probe the neutral-charged WIMP doublet models. The $O(1-10 \mathrm{MeV})$ scale energy deposition in (11) and (2) give $0 \nu \beta \beta$ searches a clear advantage over direct DM detection experiments. The latter are optimized to detect keV-scale energy depositions from the elastic scattering of WIMPs and the MeV-scale energy range is usually not reported. Moreover, albeit similar detection principles and background rates, $0 \nu \beta \beta$ experiments are more readily scaleable in mass, and the next decade is bound to produce progress not only in limiting/detecting the Majorana mass for neutrinos, but also in constraining the charged excitations of WIMPs. In what follows we consider representative models for both cases and use them to set constraints from new data released this year by EXO-200 [5] and Kamland-Zen [6] $0 \nu \beta \beta$ collaborations.

Representative Models Case A demands that the spins in the DM multiplet differ. With an eye on SUSY we take the DM particle to be a Majorana fermion $X^{0}=\chi$ (like the neutralino) and the charged excited state to be a complex scalar $X^{-}=\tilde{\tau}^{-} \equiv \tilde{\tau}$ (like the stau.) The effective Lagrangian which governs the bound state formation is of Yukawa type

$$
\mathcal{L}_{\mathrm{A}}=\bar{\chi}\left(g_{e L} \mathbb{P}_{L}+g_{e R} \mathbb{P}_{R}\right) e \tilde{\tau}^{\dagger}+\text { h.c. },
$$

with in general complex couplings $g_{e L, R}$ for the chirality projections $\mathbb{P}_{L, R}$.

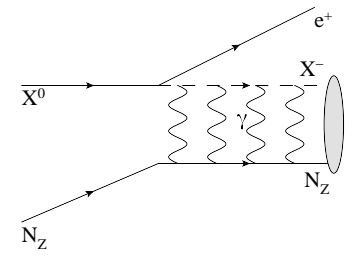

(a)

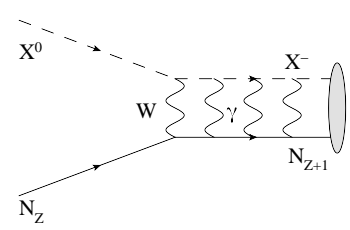

(b)
FIG. 1: Illustrations of case A (a) and case B (b). 
TABLE I: Relevant nuclei, binding energies, and exposures $M T$ for different experiments considered in this work.

\begin{tabular}{|c|c|c|c|c|}
\hline Exps. & $\begin{array}{c}\text { EXO-200/ } \\
\text { Kaml.-Zen/ } \\
\text { Xe100 }\end{array}$ & $\begin{array}{c}\text { DAMA } \\
\mathrm{NaI}(\mathrm{Tl})\end{array}$ & $\begin{array}{l}\mathrm{SNO} \\
\mathrm{NaCl}\end{array}$ & $\begin{array}{l}\text { Bore- } \\
\text { xino }\end{array}$ \\
\hline Nucleus & $\mathrm{Xe}$ & $\mathrm{I}$ & $\mathrm{Cl}$ & $\mathrm{C}$ \\
\hline$E_{b}^{(0)}(\mathrm{MeV})$ & 18.4 & 18.2 & 6.3 & 2.7 \\
\hline$M T(\mathrm{~kg}$ yr $)$ & $40 / 30 / 0.9$ & 7.5 & 1274 & $1.3 \times 10^{5}$ \\
\hline
\end{tabular}

In Case B the DM states share the same spin. For simplicity, we take both as scalars in the electroweak doublet, a real scalar $X^{0}=\tilde{\nu}^{0}$ (like a sneutrino) and a complex scalar $X^{-}=\tilde{\tau}^{-}$as before. The relevant effective Lagrangian can be written as

$$
\mathcal{L}_{\mathrm{B}}=\frac{g_{\text {eff }}}{2} W^{-\mu}\left(\partial_{\mu} \tilde{\tau}^{\dagger} \tilde{\nu}^{0}-\tilde{\tau}^{\dagger} \partial_{\mu} \tilde{\nu}^{0}\right)+\text { h.c. }+\mathcal{L}_{(4)}
$$

where $\mathcal{L}_{(4)}$ contains quartic interactions. Assuming that the sneutrino state has small admixture of sterile states, in SUSY $g_{\text {eff }}=g_{2} \cos \theta_{\tilde{\tau}}$, where $g_{2}$ is the gauge coupling of the weak interaction and $\theta_{\tilde{\tau}}$ is the mixing angle between the left and right handed stau.

Bound state properties and formation In Tab. I we list the ground state energies $E_{b}^{(0)}$ for the $X^{-}$bound states with relevant nuclei, using the homogeneous nuclear charge distribution within sphere of radius $R_{0}=$ $\sqrt{5 / 3} R_{\text {rms }}$, and $R_{\text {rms }}$ values are taken from Ref. 7]. Kinetic energies $O(100 \mathrm{keV})$ of the incoming DM and the recoiling bound state can be neglected, and the expected total visible energy $E_{\text {tot }}$ injected in the detector is given by

$$
E_{\mathrm{tot}} \approx \begin{cases}E_{b}^{(0)}-\Delta m+m_{e} & \text { Case A, } \\ E_{b}^{(0)}-\Delta m+m_{Z}-m_{Z+1} & \text { Case B. }\end{cases}
$$

In Case A the positron is emitted with an energy $E_{e}^{(n, l)}=$ $E_{b}^{(n, l)}-\Delta m-m_{e}$, where $m_{e}$ is the electron mass and $n, l$ denote the usual initial principal and orbital quantum numbers of the capture. After the positron is stopped, its annihilation with an electron yields an additional energy of $2 m_{e}$ whereas the excited $(N \tilde{\tau})^{*}$ relaxes by emission of gamma-rays. In case B no positron is emitted but the difference in nuclear mass upon the nuclear transmutation $N_{Z} \rightarrow N_{Z+1}$ becomes accessible.

For case A, the product of recombination cross section $\sigma_{A}$ with the incoming DM velocity $v$ reads,

$$
\sigma_{A} v \simeq\left(\left|g_{e L}\right|^{2}+\left|g_{e R}\right|^{2}\right) /\left(8 \pi m_{\chi}\right) \times \sum_{n, l} B_{n, l},
$$

where $B_{n, l}$ is the contribution from capture into state $(n, l)$. In the limit $\Delta m \gg m_{e}$ and $p_{\chi} \gg p_{e^{+}}$,

$$
\begin{aligned}
B_{n, l} & \simeq\left(E_{b}^{(n, l)}-\Delta m-m_{e}\right) \sqrt{\left(E_{b}^{(n, l)}-\Delta m\right)^{2}-m_{e}^{2}} \\
& \times \int d^{3} r_{1} d^{3} r_{2} \phi_{n, l}^{*}\left(\vec{r}_{1}\right) \phi_{n, l}\left(\vec{r}_{2}\right) e^{i \mu \vec{v} \cdot\left(\vec{r}_{1}-\vec{r}_{2}\right)}
\end{aligned}
$$

Here, $\phi_{n, l}$ is the wave function of the relative motion of $(N \tilde{\tau})$ with reduced mass $\mu$. For $n \leq 50$ we calculate $B_{n, l}$ explicitly by numerical solution of the Schrödinger equation. Whenever $\Delta m$ is small, capture into a multitude of states (also with $n>50$ ) is possible. Importantly, in this case we expect $\sigma_{A}$ to flow towards a semi-classical (SC) limit: when $\chi$ approaches the nucleus, a critical radius $r_{b}$ in the electromagnetic potential $V(r)$ will be reached when the transition $\chi \rightarrow \tilde{\tau}^{-}+e^{+}$becomes energetically possible, $V\left(r_{b}\right)+\Delta m+m_{e} \leq 0$. The integration over the fly-by time when $r<r_{b}$ then gives the rate for this transition to happen. In this SC limit we find for $\sum_{n, l} B_{n, l}$,

$$
\int_{|\vec{r}|<r_{b}} d^{3} \vec{r} \sqrt{(V(|\vec{r}|)+\Delta m)^{2}-m_{e}^{2}}\left(-V(|\vec{r}|)-\Delta m-m_{e}\right)
$$

Whenever, say, $n>10$ is accessible in the capture, we find a perfect agreement between the SC calculation and the explicit quantum mechanical calculation. For lighter nuclei with shallow binding the latter is the preferred choice as typically only few states contribute to the signal above the detector threshold. As expected, being an inelastic process, $\sigma_{A} v$ is largely independent of $v$, i.e. $\left\langle\sigma_{A} v\right\rangle \simeq \sigma_{A} v$.

Once $\Delta m$ and DM mass $m_{\chi}$ are chosen, the induced signal in the neutrino searches can be translated into a constraint on the combination of Yukawa couplings $\left(\left|g_{e L}\right|^{2}+\left|g_{e R}\right|^{2}\right)$. We choose to trade the latter against a constraint on the $\tilde{\tau}$ lifetime instead, or, equivalently on its decay width $\Gamma_{\tilde{\tau}}=\tau_{\tilde{\tau}}^{-1}$. In the same approximations,

$$
\Gamma_{\tilde{\tau}} \simeq \frac{\sqrt{\Delta m^{2}-m_{e}^{2}}}{4 \pi m_{\chi}}\left(\Delta m+m_{e}\right)\left(\left|g_{e L}\right|^{2}+\left|g_{e R}\right|^{2}\right)
$$

Let us now consider case B. During the capture an interconversion from a neutron $(n)$ to a proton $(p)$ takes place inside the nucleus. We use the Fermi gas model for the density and momentum distributions of $n$ and $p$ inside the nucleus $N_{Z}$. The calculation of $\sigma_{B}$ proceeds in two steps. First, we compute the fundamental process of $n \rightarrow p$ in the presence of $\tilde{\nu}^{0}$. Because of Pauli blocking, part of the binding energy is invested in elevating $p$ above the Fermi surface of $N_{Z+1}$. In a second step we again obtain the total cross section by consideration of the flyby time of $\tilde{\nu}^{0}$ in which the transition is possible. The 
result reads,

$$
\begin{aligned}
\sigma_{B} v & =\frac{g_{\mathrm{eff}}^{4} m_{p}^{2}}{8 M_{W}^{4}} \int d^{3} r \rho_{n}(\vec{r}) \int_{m_{n}}^{m_{n}+\frac{p_{n F}^{2}}{2 m_{n}}} \frac{d p_{n}^{0}}{\frac{4}{3} \pi p_{p f}^{3}} \\
& \times \sqrt{\left(-V(r)-\Delta m+p_{n}^{0}\right)^{2}-m_{p}^{2}} \sqrt{p_{n}^{0^{2}}-m_{n}^{2}} \\
& \times \theta\left(-V(r)-\Delta m+p_{n}^{0}-m_{p}-\frac{p_{p F}^{2}\left(N_{Z+1}\right)}{2 m_{p}}\right),
\end{aligned}
$$

where $\rho_{n}$ is the number density of neutron inside the target nucleus and $p_{p F}\left(N_{Z+1}\right)$ is the Fermi momentum of the $N_{Z+1}$ nucleus. The latter is determined from $m_{p}+$ $\frac{p_{p F}^{2}\left(N_{Z+1}\right)}{2 m_{p}}=m_{n}+\frac{p_{n F}^{2}\left(N_{Z}\right)}{2 m_{n}}$. For the capture on xenon, $N_{Z}=$ Xe and $N_{Z+1}=$ Cs.

This calculation is SC, and is valid for small values of $\Delta m$, when the energy release is significant relative to the typical nuclear level intervals, and many bound states are available for capture. Without detailed knowledge of the nuclear wave functions, an exact quantum mechanical calculation is not possible, although it is expected that the cross section can be dominated by a series of narrow resonances especially when $\Delta m$ is large [1, 8] .

Neutrino experiments We now come to the central part of our study - demonstrating the potential of neutrino experiments and in particular of $0 \nu \beta \beta$ searches in probing DM. As we have argued, the total energy in (5) is monochromatic with all its energy injected almost instantaneously. The signal shape is hence determined by the energy resolution $\sigma_{e}$ of the experiment. Given an exposure $M T$ the total number of events in an energy bin $\Delta E=E_{\max }-E_{\min }$ then reads,

$$
\begin{aligned}
& N_{\mathrm{tot}}=\frac{M T N_{T} \rho_{\mathrm{DM}}\langle\sigma v\rangle}{2 m_{X^{0}}} \\
& \quad \times\left[\operatorname{Erf}\left(\frac{E_{\mathrm{max}}-E_{\mathrm{tot}}}{\sqrt{2} \sigma_{e}}\right)-\operatorname{Erf}\left(\frac{E_{\mathrm{min}}-E_{\mathrm{tot}}}{\sqrt{2} \sigma_{e}}\right)\right],
\end{aligned}
$$

where $\rho_{\mathrm{DM}} \approx 0.3 \mathrm{GeV} / \mathrm{cm}^{3}$ is the local DM energy density, $N_{T}$ is the number of target nuclei per $\mathrm{kg}$ of active detector mass.

We start by considering the EXO-200 experiment [5] which has $110 \mathrm{~kg}$ fiducial mass enriched in ${ }^{136} \mathrm{Xe}$, whereas for our purpose the entire mass of Xe in the fiducial volume becomes active, and the exposure as a DM target is listed in Tab. [1 The TPC-type setup of EXO-200 allows to distinguish between events occurring at a single sites (SS) or at multiple sites (MS). The capture typically qualifies as an MS event, given the macroscopic range of produced $\gamma$-rays $(\approx 6 \mathrm{~cm}$ at $1 \mathrm{MeV}$ in LX.) We use the last bin containing 24 events above $3.5 \mathrm{MeV}$ to set a constraint which thereby probes the region $\Delta m \lesssim 15 \mathrm{MeV}$. Two complications arise: 1) part of $E_{\text {tot }}$ can be taken out of the fiducial volume by $\gamma$-rays and 2) events at SS with depositions greater than $10 \mathrm{MeV}$ are automatically discarded. We have studied both effects in a dedicated Monte Carlo (MC) analysis (to be presented in detail elsewhere) and find that this can weaken the limits by up to a factor of two, and which is taken into account in presented constraints. The results for cases A and B are shown in Fig. 2

The next experiment we consider is Kamland-Zen [6]. To obtain a conservative constraint, we use the reported spectrum from which we only subtract the background from the $2 \beta$ decay of ${ }^{136} \mathrm{Xe}$. The energy resolution is excellent so that we only need to consider the bin in which $E_{\text {tot }}$ lies including the neighboring ones (with appropriate statistical penalty) in deriving the constraint. Unfortunately, Kamland-Zen does not report a cumulative last bin like EXO-200 does. Therefore, we can only probe energy depositions below the last reported bin at $3.8 \mathrm{MeV}$. This, in turn, restricts the considered mass splittings to $\Delta m \gtrsim 14 \mathrm{MeV}$ as shown in Fig. 2. Once $\Delta m$ becomes large enough to approach the threshold, the limits become correspondingly weaker because of limited phase space and higher count rates.

Turning to small values $\Delta m \lesssim 4 \mathrm{MeV}$ we either probe energy depositions beyond $10 \mathrm{MeV}$ with heavy elements like with Xe or, alternatively, we can probe smaller (and hence reported) energy depositions using lighter nuclei. For the latter, the neutrino experiments of choice are currently the salt phase of the SNO water Cherenkov detector with a 2 ton loading of $\mathrm{NaCl}$ and the Borexino detector with its carbon-based scintillator. We set constraints using the respective event spectra reported in [9] and [10] where in the former case we subtract the solar neutrino signal. The excluded regions are shown on-top of the EXO-200 one. Finally, we note that $\Delta m \lesssim 4 \mathrm{MeV}$ is also challenged by severe limits [11] of anomalously heavy C, N, O nuclei among others. From Fig. 2 it can be seen that the limit from Borexino is comparable.

Direct DM detection experiments One of the current most sensitive direct detection experiments is XENON100. Since it is a LX detector, we can directly compare it to EXO-200 and Kamland-Zen. Using the electromagnetic background spectrum up to $3 \mathrm{MeV}$ published from a commissioning run [12], we find that constraint is at least one order of magnitude weaker. This can be traced back to higher levels of radioactive background present in XENON100.

A competitive experiment in sensitivity can be DAMA/LIBRA with its $250 \mathrm{~kg}$ of NaI scintillating crystals. A high-energy "single-hit" spectrum from 9 out of 25 crystals of a 24 day run was reported in [13]. No events above $10 \mathrm{MeV}$ were observed. A conservative constraint in case A is obtained by considering only recombination into the $1 S$ ground state of I because no rays are produced. The latter may well escape one crystal challenging a single-hit observation. For this reason we refrain from studying case B which certainly requires MC modeling. 

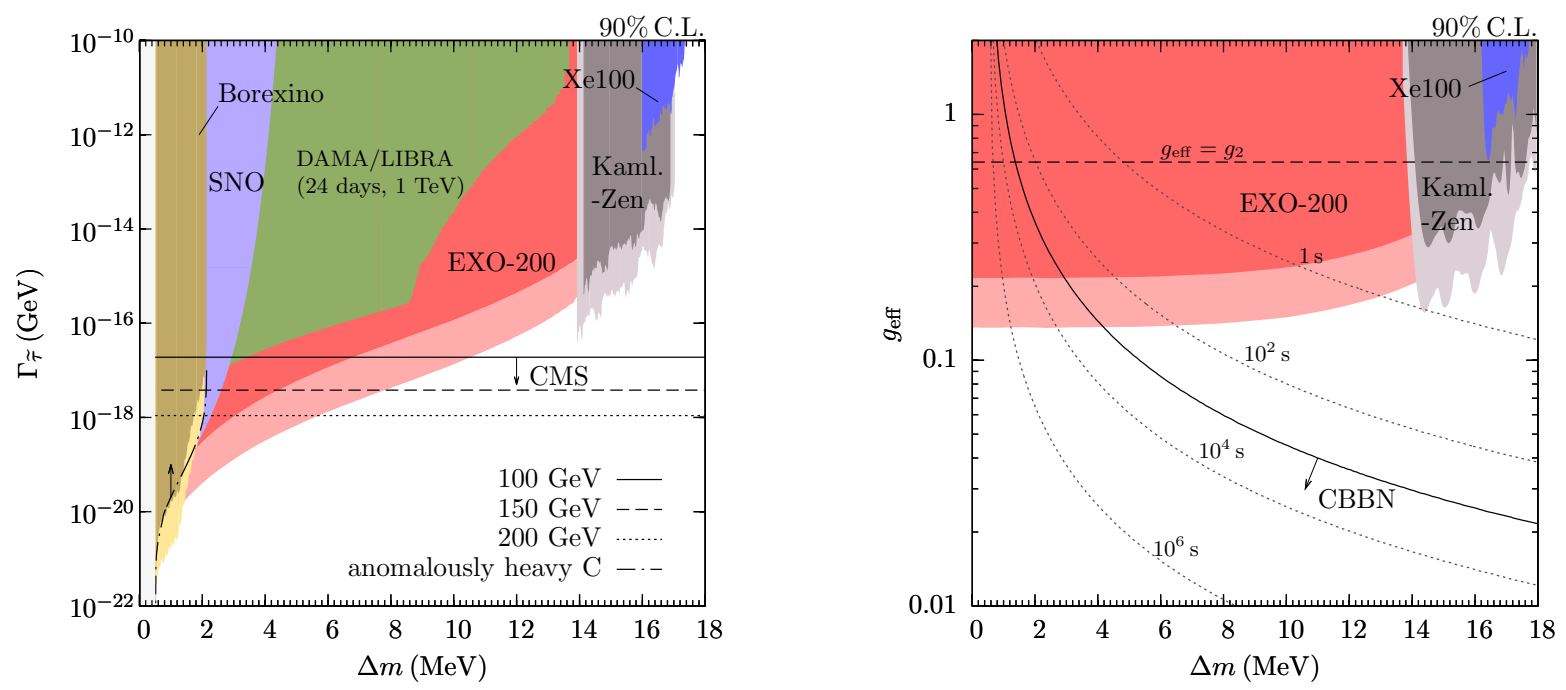

FIG. 2: Left: Constraints for "Case A" from underground rare event searches as labeled. Darker (lighter) shadings are for $m_{\chi}=1 \mathrm{TeV}(100 \mathrm{GeV})$. Also shown are lower limits from CMS searches on long-lived charged states. Right: Corresponding constraints for "Case B". The dotted lines are contours of constant $\tilde{\tau}$ lifetime; lighter shadings are for $m_{\chi}=200 \mathrm{GeV}$.

For case A we find that the constraint is superseded by the one of EXO-200. We stress that if DAMA were to present us with a high-energy spectrum of a larger exposure, a very strong result could be obtained.

Constraints from Colliders For heavy charged stable particles, the CMS group placed a very stringent bound based on the data with an integrated luminosity of $5 \mathrm{fb}^{-1}$ and a centre-of-mass energy of $7 \mathrm{TeV}[14]$. For case A we obtain a limit on $\Gamma_{\tilde{\tau}}$ from the constraint on the LHC $\tilde{\tau}$ electroweak production cross section $\sigma_{\text {production, }}$,

$$
\sigma_{\text {production }} \times \exp \left(-R \Gamma_{\tilde{\tau}} / \gamma v_{T}\right)<\sigma_{\text {constraint }},
$$

where $R$ is the transverse distance, $v_{T}$ the transverse velocity and $\gamma$ the boost factor; $\sigma_{\text {constraint we take from [14]. }}$. The lower limits on $\Gamma_{\tilde{\tau}}$ for $m_{\chi}=100 \mathrm{GeV}$ (and above) are shown in the left Fig. 22 The combined constraints rule out the entire region $\Delta m<5 \mathrm{MeV}$ as long as $m_{\chi}<210 \mathrm{GeV}$; larger values of $m_{\chi}$ are currently not constrained by CMS. In the case that the excited state is a spinor, the constraint will become stronger because the dominant production channel is s-wave, instead of pwave in the scalar case. In case B, $\tilde{\tau}$ escapes the detector because $\Gamma_{\tilde{\tau}}$ is suppressed by $\Delta m^{5} / m_{W}^{4}$. The CMS constraint is then: $m_{\tilde{\nu}}>210 \mathrm{GeV}$. Closely related searches are also performed by ATLAS, but specialized to specific SUSY cases, and we do not use it here.

Finally, for lifetimes $\tau_{\tilde{\tau}} \gtrsim 10^{3} \mathrm{~s}$ a primordial abundance of $\tilde{\tau}^{-}$(which is expected to be similar to that of $\tilde{\nu}^{0}$ since $\Delta m / m_{\tilde{\nu}} \ll 1 \%$ ) will have a large effect on the primordial light elements; see [4] and references therein. The approximate constraint is labeled "CBBN".

Outlook We have shown that rare underground event searches can be used to obtain strong limits on charged excited states of DM with mass splittings $\Delta m \lesssim 20 \mathrm{MeV}$. Significant improvements on these bounds can be expected from nearly all upcoming $0 \nu \beta \beta$ searches, see [15] and references therein, as well as from future data sets of Kamland-Zen and EXO-200. In some cases great sensitivity is already experimentally established but not published. For example, Kamland-Zen has not reported its count rate above $3.8 \mathrm{MeV}$. Presumably, the associated constraint can be the dominant one throughout the entire considered region in $\Delta m$. Likewise, DAMA has shown a fraction of its high energy spectrum with an exposure of 24 days - whereas more than 100 times of this is available. We urge these collaborations to present this data. Acknowledgements We thank Drs. J. Chen, M. Marino, A. Ritz, N. Toro for useful discussions.

[1] M. Pospelov and A. Ritz, Phys. Rev. D 78, 055003 (2008) arXiv:0803.2251 [hep-ph]].

[2] M. Cirelli, N. Fornengo and A. Strumia, Nucl. Phys. B 753, 178 (2006) hep-ph/0512090.

[3] A. Andrea, talk at the 4th International Fermi Symposium, October 2012, Monterrey, California.

[4] M. Pospelov and J. Pradler, Ann. Rev. Nucl. Part. Sci. 60, 539 (2010) arXiv:1011.1054 [hep-ph]].

[5] M. Auger et al. [EXO Collaboration], Phys. Rev. Lett. 109, 032505 (2012) arXiv:1205.5608 [hep-ex]].

[6] [KamLAND-Zen Collaboration], Phys. Rev. C 85, 045504 (2012) arXiv:1201.4664 [hep-ex]].

[7] I. Angeli, Atom. Data Nucl. Data Tabl. 87, 185 (2004).

[8] Y. Bai and P. J. Fox, JHEP 0911, 052 (2009) arXiv:0909.2900 [hep-ph]].

[9] B. Aharmim et al. [SNO Collaboration], Phys. Rev. C 
72, 055502 (2005) nucl-ex/0502021.

[10] G. Bellini et al. [Borexino Collaboration], Phys. Rev. D 85, 092003 (2012) arXiv:1203.6258 [hep-ex]].

[11] T. K. Hemmick et al., Phys. Rev. D 41, 2074 (1990).

[12] E. Aprile et al., Phys. Rev. D 83, 082001 (2011) [Erratum-ibid. D 85, 029904 (2012)] arXiv:1101.3866 [astro-ph.IM]].
[13] R. Bernabei et al., Eur. Phys. J. C 62, 327 (2009).

[14] S. Chatrchyan et al. [CMS Collaboration], Phys. Lett. B 713, 408 (2012) arXiv:1205.0272 [hep-ex]].

[15] S. R. Elliott, Mod. Phys. Lett. A 27, 1230009 (2012) arXiv:1203.1070 [nucl-ex]]. 